\title{
Gamma-ray observations of solar flares from August 2001 to November 2003: SONG experiment onboard CORONAS-F satellite results.
}

\author{
Irina N. Myagkova ${ }^{1}$, S. N. Kuznetsov ${ }^{1}$, V. G. Kurt ${ }^{1}$, E.A. Murav'eva ${ }^{1}$, \\ B. Yu. Yushkov ${ }^{1}$ and K. Kudela ${ }^{2}$ \\ ${ }^{1}$ Skobeltsyn Institute of Nuclear Physics, Moscow State University, Moscow, Russia \\ email: irina@srd.sinp.msu.ru \\ ${ }^{2}$ Institute of Experimental Physics, Slovak Academia of Science, Kosice, Slovakia \\ email: kkudela@upjs.kosice.sk.ru
}

\begin{abstract}
We present the catalog of data for 27 gamma-ray flares detected above $500 \mathrm{keV}$ by the gamma-ray spectrometer SONG (Solar Neutrons and Gamma-Rays) onboard the Russian CORONAS-F satellite. These gamma-ray flares were collected during the period from August 14, 2001 till November 31, 2003 covering the latter half of the 23rd solar sunspot cycle.
\end{abstract}

\section{Introduction}

The observations of solar flare gamma-emission provide the most direct information about the charged particle acceleration in solar flares (e.g. Ramaty, Kozlovsky \& Suri 1977). During last four solar cycles some hundreds of solar flares generated gammaemission were detected in different experiments, but the statistics of gamma-ray flares obtained during the 23 cycle was significantly poorer than in earlier cycles (especially before the extreme intensive solar flares in October - November, 2003). So new experimental data about solar gamma-emission of solar flares are important.

\section{SONG experiment and its results}

The CORONAS-F satellite was launched on July 31, 2001. It has a circular orbit with altitude about $500 \mathrm{~km}$ and 82.5 degree inclination. The detector part of SONG consists of $\mathrm{CsI}(\mathrm{Tl})$ crystal of diameter $20 \mathrm{~cm}$ and height $10 \mathrm{~cm}$ surrounded by the active anticoincidence plastic shielding $2 \mathrm{~cm}$ thickness (Kuznetsov et al. 2003). In this work we use data about gamma-emission with the energies $0.5-200 \mathrm{MeV}$. Temporal resolution for gamma-emission is $4 \mathrm{~s}$. The background, caused by the local gamma-rays produced by the interactions of CR either with the instrument, satellite body or with the atmosphere and bremstrahlung of relativistic electrons of radiation belts), was carefully substructed before the flare emission analysis.

During the first 2.5 years CORONAS-F fly more than fifty flares with HXR- emission (more then $50 \mathrm{keV}$ ) were detected by SONG-instrument. In 27 flares the gamma- emission with the energy more then $500 \mathrm{keV}$ was detected (see Table 1). Gamma- emission with energies higher than $60 \mathrm{MeV}$ was detected in the flares 25/08/2001, 28/10/2003 and $04 / 11 / 2003$. During the maximum and the decay phase of 23 solar cycle solar gamma-emission are measured in the experiments on board RHESSI (Lin et al. 2002) and CORONAS-F satellites (Kuznetsov et al. 2003). In the Table 1 we show whether 


\begin{tabular}{|c|c|c|c|c|c|c|c|c|}
\hline $\mathrm{N}$ & $\mathrm{dd} / \mathrm{mm} / \mathrm{yy}$ & UT & Class/Ball & Coordinates & RHESSY & $\mathrm{E}, \mathrm{MeV}$ & $\mathrm{p}$ & $\mathrm{e}$ \\
\hline 1 & $25 / 08 / 01$ & $16: 29-16: 38$ & $\mathrm{X} 5.3 / 3$ & S17E34 & - & $60-100$ & No enh. & no enh. \\
\hline 2 & 05/09/01 & $14: 27-14: 29$ & M6.0/2' & N15W31 & - & $1.3-4$ & No data & No data \\
\hline 3 & 09/09/01 & $15: 11-15: 13$ & $\mathrm{M} 3.4 / 1 \mathrm{~N}$ & S17E03 & - & $.5-1.3$ & No enh. & No enh. \\
\hline 4 & $19 / 10 / 01$ & 16:23-ERB & $\mathrm{X} 1.6 / 2 \mathrm{~B}$ & N15W29 & - & $1.3-4$. & 3 & 17 \\
\hline 5 & $11 / 12 / 01$ & 08:04-08:08 & $\mathrm{X} 2.8 / \mathrm{SF}$ & N16E41 & - & $7-15$ & 40 & 33 \\
\hline 6 & $20 / 02 / 02$ & 06:08-06:11 & M5.1/1N & N12W72 & No & $4-7$ & 140 & 7 \\
\hline 7 & $20 / 05 / 02$ & $15: 25-15: 29$ & $\mathrm{X} 2.1 / 2 \mathrm{~N}$ & S21E65 & No & $7-15$ & No data & No data \\
\hline 8 & $17 / 07 / 02$ & 07:10-07:13 & M8.5/1B & N22W17 & No & $1.3-4$ & 1200 & 2000 \\
\hline 9 & $20 / 07 / 02$ & 21:08-21:29 & $\mathrm{X} 3.3 /-$ & - & Yes & $0.5-1.3$ & 20 & 700 \\
\hline 10 & $20 / 08 / 02$ & $08: 24-08: 26$ & M3.4/1B & S10W38 & Yes & $4-7$ & 60 & 600 \\
\hline 11 & $21 / 08 / 02$ & 01:38-01:42 & M1.4/SF & S10W36 & Yes & $0.5-1.3$ & No enh. & No enh. \\
\hline 12 & $21 / 08 / 02$ & 05:31-05:33 & $\mathrm{X} 1.0 / 1 \mathrm{~B}$ & S12W51 & No & $4-7$ & No enh. & No enh. \\
\hline 13 & $24 / 08 / 02$ & 00:58-01:03 & $\mathrm{X} 3.4 / 1 \mathrm{~F}$ & S12W51 & Partly & $4-7$ & 200 & 700 \\
\hline 14 & $30 / 08 / 02$ & $13: 27-13: 29$ & $\mathrm{X} 1.5 / \mathrm{SN}$ & N15E74 & Yes & $4-7$ & No enh. & No enh. \\
\hline 15 & $26 / 04 / 03$ & 03:03-03:04 & $? 2.1 / \mathrm{SN}$ & S25W34 & Yes & $1.3-4.0$ & No enh. & No enh. \\
\hline 16 & $26 / 04 / 03$ & 08:05-08:07 & M2.0/- & - & Yes & $4-7$ & No enh. & No enh. \\
\hline 17 & $27 / 05 / 03$ & 23:02-23:09 & $\mathrm{X} 1.3 / 2 \mathrm{~B}$ & S07W17 & Yes & $4-7$ & No data & No data \\
\hline 18 & $28 / 05 / 03$ & $00: 21-00: 29$ & $\mathrm{X} 3.6 / 3 \mathrm{~B}$ & S07W17 & Yes & $4-7$ & No data & No data \\
\hline 19 & $29 / 05 / 03$ & 00:59-01:07 & $\mathrm{X} 1.2 /-$ & - & Yes & $4-7$ & No data & No data \\
\hline 20 & $23 / 10 / 03$ & 08:17-08:43 & $\mathrm{X} 5.4 / 1 \mathrm{~B}$ & S21E88 & No & $4-7$ & 100 & 150 \\
\hline 21 & $28 / 10 / 03$ & 11:02-11:13 & $17.2 / 4 \mathrm{~B}$ & S16E08 & Partly & $60-100$ & 8000 & 15000 \\
\hline 22 & $29 / 10 / 03$ & $20: 38-20: 55$ & $\mathrm{X} 10 / 2 \mathrm{~B}$ & S15W02 & Yes & $4-7$ & 500 & 13000 \\
\hline 23 & $03 / 11 / 03$ & 01:17-01:29 & $\mathrm{X} 2.7 / 2 \mathrm{~B}$ & N10W83 & Yes & $0.5-1.3$ & No data & No data \\
\hline 24 & $04 / 11 / 03$ & $19: 32-19: 57$ & $\mathrm{X} 28 / 3 \mathrm{~B}$ & S19W83 & Partly & $100-200$ & 1000 & 1800 \\
\hline 25 & $17 / 11 / 03$ & 08:59- 09:05 & $\mathrm{M} 4.2 / 1 \mathrm{~N}$ & S01E33 & Partly & $1.3-4$ & No enh. & No enh. \\
\hline 27 & $20 / 11 / 03$ & 08:04- 08:18 & M9.5/- & - & Yes & $0.5-1.3$ & 45 & 100 \\
\hline
\end{tabular}

Table 1. Solar flares detected by SONG instrument (CORONAS-F)

RHESSY data available or not during flare detected by SONG. We can see that the most of flares were detected in both experiments. This gives us the opportunity to compare obtained data for better understanding of processes during solar flares. Besides usual solar flare parameters we show the value of solar proton (1-5 MeV) end electron (300-600 keV) flux enhancements in $1 /\left(\mathrm{cm}^{* *} 2 \mathrm{~s} \mathrm{sr}\right)$ detected on board CORONAS-F by after each flare. This information is important for the Earth's radiation environment and space weather studies.

First two and a half years SONG experiment on board CORONAS-F not only illustrated its capability of detection of solar gamma-emissions, but gave us the valuable information for studying of flare processes. Obtained SONG gamma-flares catalog should be very useful for future statistical and correlative studies of solar flare intrinsic properties and solar particle events probability.

\section{Acknowledgements}

We would like to acknowledge J. Rojko and J. Balaz for work on developing SONG electronics, and Slovak grant agency VEGA, project 4/4064 for support.

\section{References}

S. N. Kuznetsov, K. Kudela, I. N. Myagkova \& B. Yu. Yushkov 2003 In Solar variability as an Input to the Earth's Environment (ed. A. Wilson). pp. 683-686. ESA SP-535.

Lin R.P. et al. 2002 Solar Phys. 210, 3-32.

Ramaty R, Kozlovsky B. \& Suri A.N. 1977 Astrophys. J. 214, 617-625. 